\title{
Non-Boltzmann stationary distributions and non-equilibrium relations in active baths
}

\author{
Aykut Argun*1, Ali-Reza Moradi ${ }^{1,2,3}$, Erçağ Pinçe1, Gokhan Baris Bagci ${ }^{4}$, \\ Alberto Imparato ${ }^{5}$ and Giovanni Volpe ${ }^{1,6}$ \\ ${ }^{1}$ Department of Physics, University of Gothenburg, 41296 Gothenburg, Sweden \\ ${ }^{2}$ Department of Physics, University of Zanjan, PO Box 45195-313, Zanjan, Iran \\ ${ }^{3}$ Optics Research Center, Institute for Advanced Studies in Basic Sciences, PO Box 45137-66731, Zanjan, Iran \\ ${ }^{4}$ Department of Materials Science and Nanotechnology Engineering, TOBB University of Economics and Technology, 06560 Ankara, Turkey \\ ${ }^{5}$ Department of Physics and Astronomy, University of Aarhus Ny Munkegade, Building 1520, DK-8000 Aarhus C, Denmark \\ ${ }^{6}$ UNAM -- National Nanotechnology Research Center, Bilkent University, Ankara 06800, Turkey \\ *Corresponding author: argunaykut@gmail.com
}

\begin{abstract}
The presence of active noise generated by motile bacteria results in the violation of Boltzmann distribution. Therefore, non-equilibrium relations become invalid active baths. Yet these relations can be recovered by introducing effective potentials.

OCIS codes: (000.6850) Thermodynamics; (140.7010) Laser trapping
\end{abstract}

Most natural and engineered processes, such as biomolecular reactions, protein folding, and population dynamics, occur far from equilibrium and, therefore, cannot be treated within the framework of classical equilibrium thermodynamics [1]. Here, we experimentally study how some fundamental thermodynamic quantities and relations are affected by the presence of the non-equilibrium fluctuations associated with an active bath [2]. We show, in particular, that, as the confinement of the particle increases, the stationary probability distribution of a Brownian particle confined within a harmonic potential becomes non-Boltzmann, featuring a transition from a Gaussian distribution to a heavy-tailed distribution. Because of this, non-equilibrium relations (e.g. Jarzynski equality [3], Crooks fluctuation theorem [4]) cannot be applied. We show that these relations can be restored by using the effective potential associated with the stationary probability distribution. We corroborate our experimental findings with theoretical arguments[5].
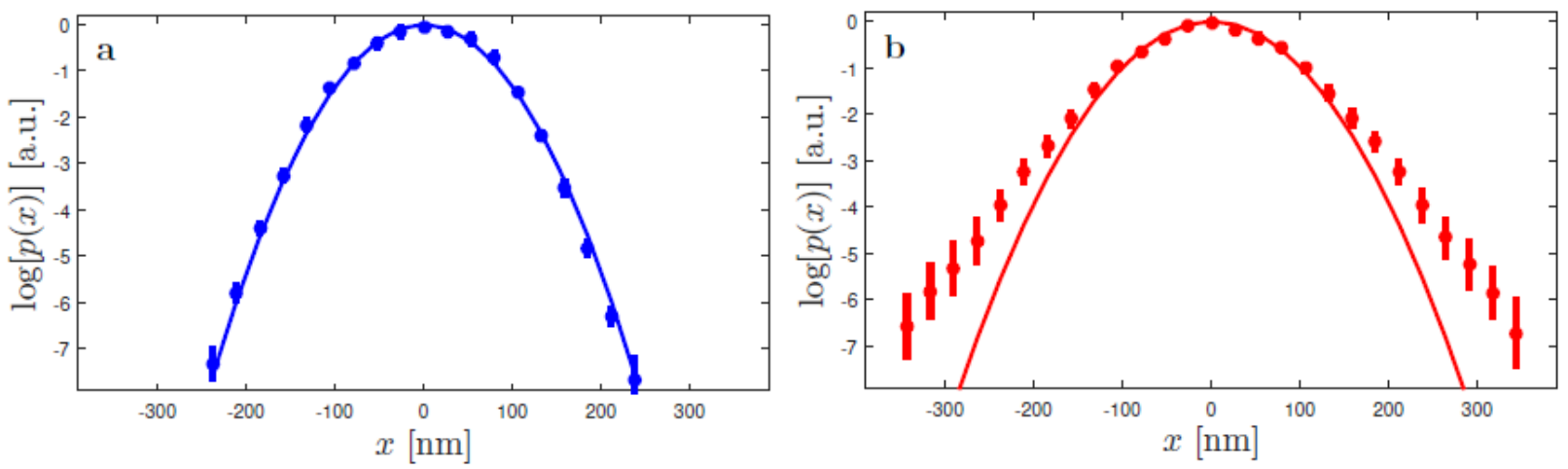

Non-Boltzmann statistics in an active bath. a. The experimental probability distribution of the position of a particle (symbols) held in a harmonic potential in a thermal bath is Gaussian (line, corresponding to the theoretical prediction for a particle held in a harmonic potential. This corresponds to the Boltzmann statistics associated with the harmonic potential. b. However, the experimental position distribution (symbols) in an active bath deviates from a Gaussian (best fit represented by the line) and features heavy tails. This is a signature that the statistics in the active bath are non-Boltzmann and that the behavior of a particle in an active bath is qualitatively different from that in a thermal bath, i.e. it cannot correspond to a different (higher) effective temperature. The data are obtained from 200000 position measurements 
In conclusion, we have shown that in an active bath, when a system is confined within a potential whose length scale is comparable or smaller than the characteristic length scale associated with the active noise, non-Boltzmann statistics emerge. The introduction of an effective temperature is not sufficient to provide a framework within which to describe such systems. Such framework can be instead provided by the use of an effective potential related to the system stationary distribution. This effective potential plays the same role as the mechanical potential; in particular, the stochastic work and heat can be obtained from it, and such quantities turn out to satisfy the same fluctuation relations as the corresponding quantities in the passive bath case. We have exemplified these results with a series of experiments using an optically trapped particle in an active (bacterial) bath. Since active matter plays a central role in many systems, including very importantly living systems, our findings pose some significant limitations to the possibility of applying non-equilibrium fluctuation relations in their classical formulation to study this broad and valuable class of systems, pointing to the need for alternative approaches that explicitly model the presence of nonthermal fluctuations.

[1] M. Cates, "Diffusive transport without detailed balance in motile bacteria: does microbiology need statistical physics?", Reports on Progress in Physics 75 (4), 042601 (2012).

[2] Xiao-Lun Wu and Albert Libchaber, "Particle Diffusion in a Quasi-Two-Dimensional Bacterial Bath", Physical Review Letters 84, 3017 (2000).

[3] C. Jarzynski, "Nonequilibrium equality for free energy differences", Physical Review Letters 78 (14), 2690 (1997).

[4] G. E. Crooks, "Entropy production fluctuation theorem and the nonequilibrium work relation for free energy differences", Physical Review E 60 (3), 2721 (1999)

[5] A. Argun, A.R. Moradi, E. Pinçe, G.B. Bagci and Giovanni Volpe, "Experimental evidence of the failure of the Jarzynski equality in active baths", arXiv:1601.01123 (2016) 